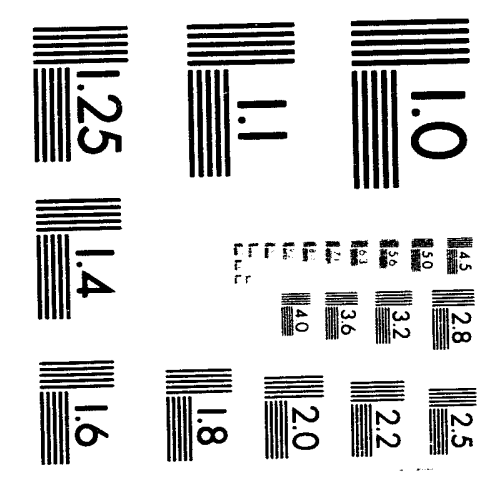



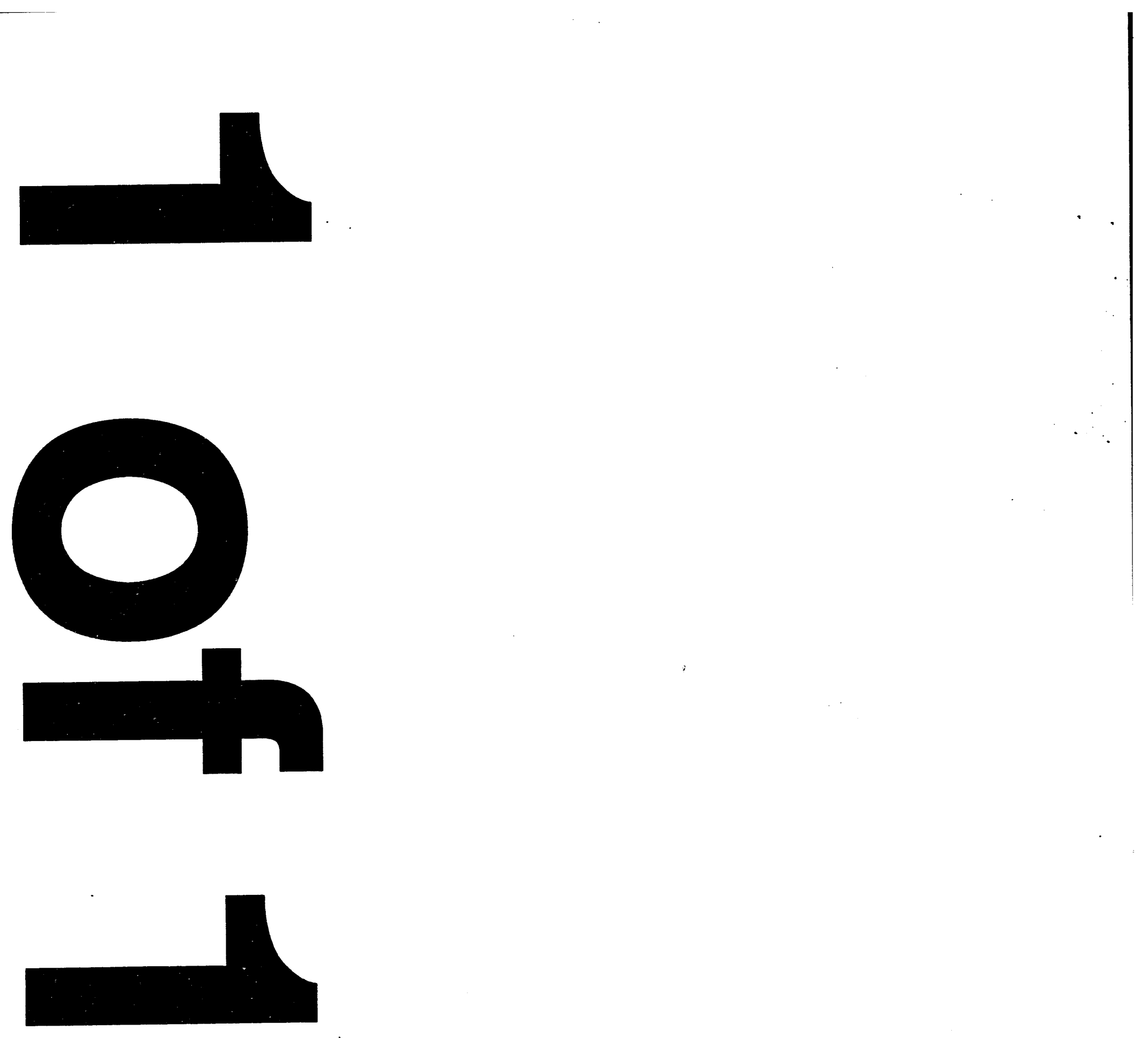


\title{
ADSORPTION AND DESORPTION STUDIES OF CESIUM ON SAPPHIRE SURFACES
}

\author{
Kevin R. Zavadil \\ Sandia National Laboratories \\ P.O. Box 5800 \\ Albuquerque, NM 87185-5800 \\ (505) $845-8442$
}

\author{
Judith L. Ing \\ AEA Technology (TOPAZ-II Project) \\ 901 University Blvd. SE \\ Albuquerque, NM 87106-4439
}

(505) 272-7409

\begin{abstract}
The adsorption/desorption characteristics of Cs on sapphire surfaces have been studied using a combination of surface analytical techniques. An approximate initial sticking coefficient for Cs on sapphire has been measured using a reflection mass spectrometric technique and has been found to be 0.9 . Thermal Desorption Mass Spectrometry (TDMS) and Auger Electron Spectroscopy (AES) have been used to verify that a significant decrease in sticking coefficient occurs as ce coverage reaches a critical submonolayer value. TDMS analysis demonstrates that $\mathrm{Cs}$ is stabilized on a clean sapphire surface at temperatures (1200 K) in excess of the temperatures experienced by sapphire in a TOPAZ-II thermionic fuel element (TFE). Surface contaminants on sapphire can enhance $\mathrm{Cs}$ adsorption relative to the clean surface. $\mathrm{C}$ contamination eliminates the high temperature state of $\mathrm{Cs}$ desorption found on clean sapphire but shifts the bulk of the Cs desorption from 400 to $620 \mathrm{~K}$. Surface $\mathrm{C}$ is a difficult contaminant to remove from sapphire, requiring annealing temperatures in excess of $1400 \mathrm{~K}$. Whether Cs is stabilized on sapphire in a TFE environment will most likely depend on the relationship between surface contamination and surface structure.
\end{abstract}

\section{INTRODUCTION}

Sapphire is used as an electrical insulator in thermionic fuel elements (TFEs) because of its chemical inertness, electrical resistance and high thermal conductivity. In a TOPAZ-II TFE, sapphire is subjected to continuous Cs exposure at elevated temperatures $(900 \mathrm{~K})$. The lack of chemical reaction between $\mathrm{Cs}$ and bulk sapphire has been previously reported by Higgins (1966). To date, no work has been published detailing the interaction of Cs at the surface of sapphire at elevated temperatures. The interaction of Cs at the sapphire surface becomes an issue when considering the surface conductivity of an insulator.

The interaction of Cs at a sapphire surface is expected to be dependent on the both the surface composition and structure. Chang (1971) was one of the first investigators to describe the impact of thermal annealing on both composition and structure of several low miller index surfaces of sapphire. Chang's results show that considerable surface temperatures $(>1300 \mathrm{~K}$ ) are required to remove contaminant C. The work of Hamza and Balooch (1992) indicates that the minimum temperature required for $\mathrm{C}$ removal will vary considerably between different crystallographic orientations. These high temperature treatments also induce surface reconstruction and result in the formation of equilibrium surface structures that have atomic arrangements that are significantly different from those of the original orientation. The work of Gautier et al. (1991) shows that the formation of an equilibrium structure for the (0001) surface actually involves a series of intermediate structures. As a first step, we have focused our efforts on understanding how Cs adsorbs onto and desorbs from randomly oriented sapphire surfaces that best approximate materials used in a TFE environment.

\section{EXPERIMENTAL}

Cs adsorption/desorption studies were conducted in a joint turbomolecular and titanium sublimation pumpec vacuum chamber maintained at a base pressure of $5 \times 10^{-10}$ torr. Auger Electron Spectroscopy (AES) was conducted with a single pass cylindrical mirror analyzer (Physical Electronics 12-155) using a 1 to $2 \mathrm{keV}$ primary electron beam with a sample current of less than $1 \mu \mathrm{A} / \mathrm{mm}^{2}$. Mass spectrometric measurements were made using a 


\section{DISCLAIMER}

This report was prepared as an account of work sponsored by an agency of the United States Government. Neither the United States Government nor any agency thereof, nor any of their employees, makes any warranty, express or implied, or assumes any legal liability or responsibility for the accuracy, completeness, or usefulness of any information, apparatus, product, or process disclosed, or represents that its use would not infringe privately owned rights. Reference herein to any specific commercial product, process, or service by trade name, trademark, manufacturer, or otherwise does not necessarily constitute or imply its endorsement, recommendation, or favoring by the United States Government or any agency thereof. The views and opinions of authors expressed herein do not necessarily state or reflect those of the United States Government or any agency thereof. 
quadrupole filter with an electron impact ionization source (UTI 100C). A Cs getter (SAES) mounted on a low current feedthrough served as a high purity Cs source. The dispenser was mounted approximately $13 \mathrm{~mm}$ from the sapphire surface to ensure that most of the Cs released from the source had a high probability of first interaction with the sapphire surface. The mass spectrometer and the getter were oriented at $45^{\circ}$ with respect to one another to facilitate detection of reflected Cs from the sapphire surface during Cs deposition.

Randomly oriented, single crystal sapphire surfaces (Goodfellow) were used as deposition substrates in these experiments. The crystals used were $9.5 \mathrm{~mm}$ in diameter, $0.3 \mathrm{~mm}$ thick, and had a $1 \mu \mathrm{m}$ surface finish. Crystals were ultrasonically cleaned in de-ionized water, mounted onto the vacuum manipulator and rinsed with acetone prior to vacuum introduction. The crystal could be indirectly, resistively heated by mounting it onto a Ta mesh. A ceramic adhesive (Aremco 569) was used to bond the mesh to the back surface of the crystal. The mesh was terminated with a set of Ta tabs crimped and spot-welded to the ends. A pair of tungsten leads ( $0.5 \mathrm{~mm}$ diameter) were spot-welded to the Ta tabs and fixed to the copper pins of a high current feedthrough. The length of the W leads and the dimension of the Ta mesh were adjusted to ensure maximum power dissipation across the mesh when a DC current was passed through this structure. This mount structure was also sized to keep it out of line-of-sight of both the Cs source and the mass spectrometer. Sample temperatures were monitored with a W/WRe thermocouple bonded to the back of the crysta!. Controlled temperature ramps of $5 \mathrm{~K} / \mathrm{sec}$ with maximum temperatures of $1500 \mathrm{~K}$ could be achieved using this method. A temperature programmer (RHK, TM310) was used to voltage program a DC power supply to produce the thermal ramp for Thermal Desorption Mass Spectrometry (TDMS). We assume that the temperature at the back surface of the crystal reasonably approximates the front surface temperature given the crystal thickness and the modest ramp rates.

\section{RESULTS AND DISCUSSION}

\section{Surface Cleaning}

The interaction between Cs and sapphire is expected to be dependent on the composition and structure of the sapphire surface. Attention must therefore be given to the cleaning of the sapphire crystal prior to initiating adsorption/desorption studies. The initial sapphire surface is characterized by moderate levels (20 atomic percent) of surface carbon contamination. Figure la shows a derivative mode AES spectrum for sapphire prior to thermal

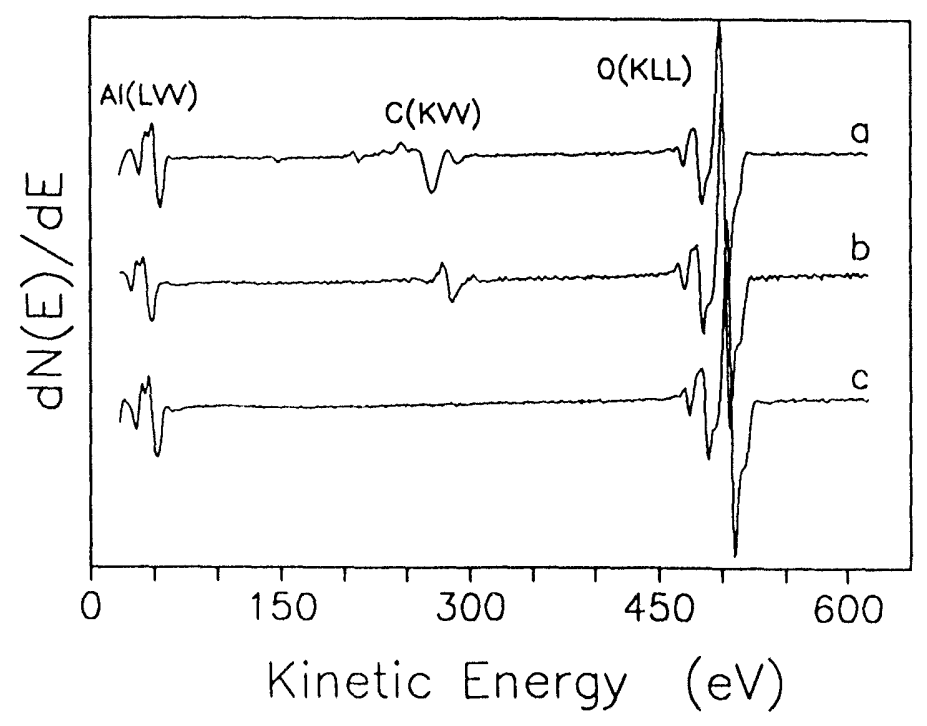

FIGURE 1: Derivative Mode AES Spectra for Sapphire Annealed to $800 \mathrm{~K}$ (a), $1400 \mathrm{~K}$ (b), and $1500 \mathrm{~K}$ (c). 
annealing. The $\mathrm{Al}(\mathrm{LVV})$ and $\mathrm{O}(\mathrm{KLL})$ transitions are visible at 48 and $510 \mathrm{eV}$, respectively. Some surface charging is implicit in these measured energies due to the insulating nature of sapphire. Sample currents of less than $1 \mu$ $\mathrm{A} / \mathrm{mm}^{2}$ were used to minimize charging. The $\mathrm{C}(\mathrm{KVV})$ transition is also visible at $270 \mathrm{cV}$. The carbon results from adventitious adsorption of organics from sample handling, atmospheric exposure, and solution pretreatment. Contaminant carbon levels can be reduced by annealing the sample to $1400 \mathrm{~K}$, but cannot be completely removed, as shown in Figure $\mathrm{lb}$. Elevated thermal treatment of the sapphire appears to produce a reduced state of carbon based on the change to a more symmetric derivative lineshapc. As shown in Figure Ic, thermal annealing of the surface to $1500 \mathrm{~K}$ is required to remove this reduced state of carbon to below AES detection limits (<1 atomic \%). These results are consistent with the findings of Chang (1971) who demonstrated that 1300 to $1500 \mathrm{~K}$ annealing was required for carbon removal and formation of equilibrium structures on various low miller index sapphire surfaces. Our annealing protocol is more stringent than the $1300 \mathrm{~K}$ anneal found to suffice for the (0001) surface by Hamza and Balooch (1992) and may be an indication of the greater energetics for our presumably more disordered surface.

\section{Cs Adsorntion}

A limited quantity of Cs will adsorb onto an atomically clean sapphire surface at surface temperatures of 310 io $320 \mathrm{~K}$. Figure $2 \mathrm{~b}$ shows the time dependence of the componcs:t of Cs flux reflected from a clean sapphire surface during Cs deposition measured as ion current using the mass spectrometer. The dispenser has been activated by stepping the current to $4.3 \mathrm{~A}$. Cs will not be released until the dispenser has warmed to some temperature and, as a result, no Cs signal is detected for the first 40 seconds of source heating. After 40 seconds, a small rise in the Cs signal is detectable from a background level of 0.08 to $0.18 \mathrm{nA}$. The sigmoid shape on the leading edge of the current rise is presumably due to the thermal time constant for the source. The signal level stays relatively constant until 95 seconds, after which a sudden increase is observed. Beyond 95 seconds, the signal asymptotically approaches a limiting value with increased time. Current to the source was shut off at 148 seconds and the Cs signal responds by exponentially decaying to the background level over a 50 second period. The fact that this varying signal response with time is predominantly a function of the rate of Cs adsorption on the sapphire surface can be demonstrated by conducting two control experiments: 1) monitoring the Cs signal with the crystal removed to a position out of line-of-sight to the source and 2) monitoring the Cs signal with the crystal in an optimum deposition position after extensive pre-exposure to Cs. The results of these two experiments are shown in Figure 2a

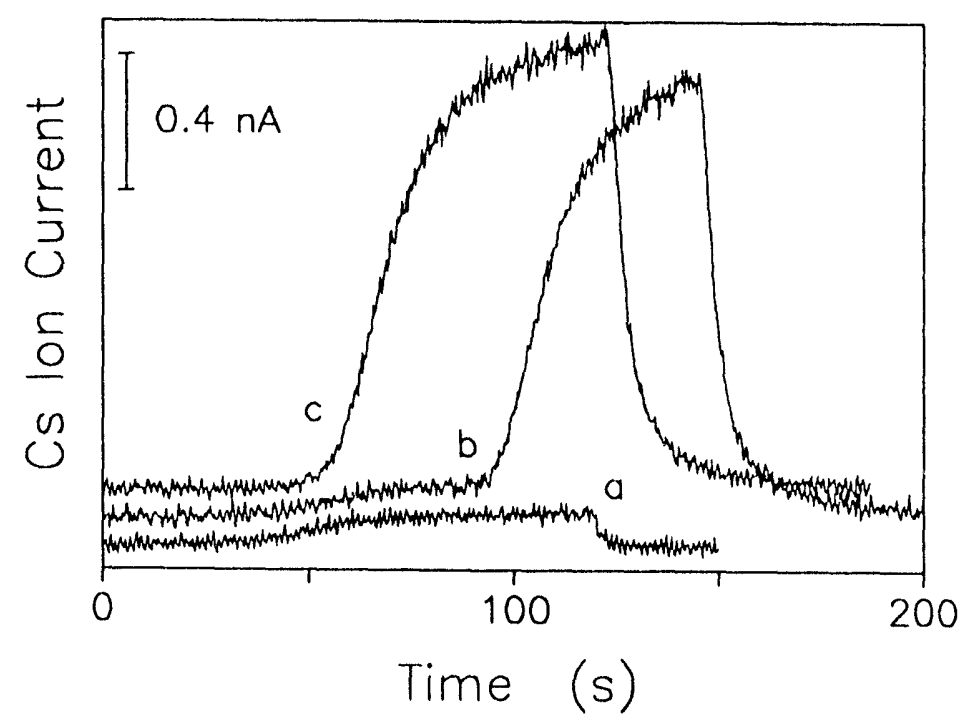

FIGURE 2: Reflected Cs Ion Current versus Deposition Time: a) System Response - Sample Removed From Lineof-Sight Position with respect to the Cs Source, b) Clean Sapphirc Response, and c) Cs-saturated Sapphire Response. 
and $2 \mathrm{c}$, respectively. The process of heating the source from room temperature to a $\mathrm{Cs}$ release temperature has been repeated for these experiments so as to provide a consistent time frame for observation. Figure 2a represents the background response of the system during Cs deposition. This trace shows that the getter starts to release Cs after 40 seconds of heating with an increase in signal from the $0.08 \mathrm{nA}$ background level. The signal increases and stabilizes at a maximum value of $0.15 \mathrm{nA}$. The signal rapidly decays with source shut-off at 110 seconds. Figure $2 \mathrm{c}$ represents the Cs-saturated response of the crystal to extended deposition. This trace shows Cs release at 40 seconds followed by a rapid rise in signal, an asymptotic approach to a maximum level, and exponential decay with source shut-off. The delayed response of the source in Figure $2 \mathrm{c}$ is predominantly due to the time constant for fully heating the getter.

The data of Figure 2 provides some valuable insight into the adsorption process of Cs on sapphire at room temperature. The fact that a Cs signal, greater than the background signal, is detectable as the source reaches a release temperature indicates that the initial sticking coefficient (or adsorption probability) is less than unity. Comparison of Cs signal during adsorption to the saturated signal level indicates that this initial sticking coefficient, although less than unity, is quite high and can be estimated to be 0.9 . This value represents relatively facile initial adsorption. It is important to note that this value for the sticking coefficient is a time averaged estimate. It is conceivable that the sticking coefficient is actually varying with Cs coverage and any indication of this in the reflected Cs signal is masked by the thermal response function of the getter. The rapid increase in signal observed at 95 seconds signifies a sudden decrease in the sticking coefficient at some critica! Cs coverage. The rate of change in the ion current after this sudden signal increase is discernibly greater than that observed for the postsaturation trace in a similar time frame. This difference indicates that $\mathrm{Cs}$ adsorption continues at a greatly attenuated rate for times in excess of 95 seconds.

The shape of the uptake curve of Cs by a clean sapphire surface can be predicted from the data of Figure 2 . Specifically, this curve should be the integral of the difference of the post-saturation and the pre-saturation curves. The resulting curve should have an approximate limiting logarithmic shape. This prediction can be verified by conducting the TDMS experiment for varying Cs exposure. Figure 3 shows the results of the TDMS experiment, where the measured Cs ion current has been integrated and plotted as a function of relative Cs dose. Cs exposure has been expressed in terms of dose as opposed to time because flux is not linear with time due to the thermal response time of the getter. We can calculate a dose value by integrating the area under the post-saturated response

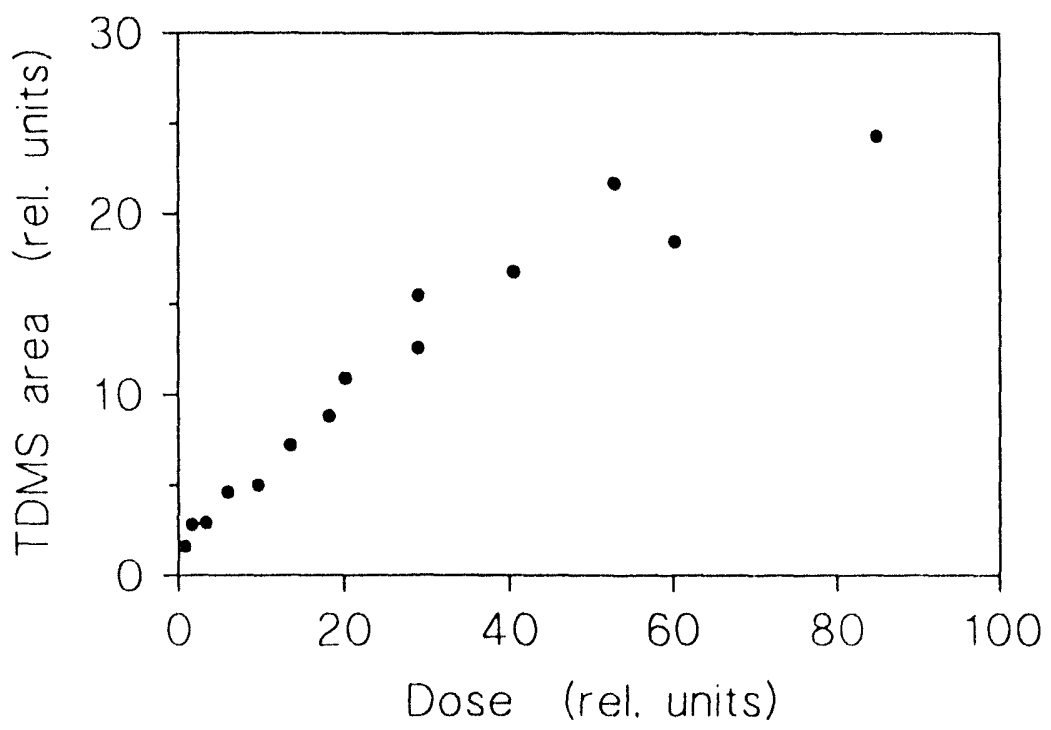

FIGURE 3: The Variation in Cs Surface Concentration Expressed in Total TDMS Area with Cs Exposure in Terms of Relative Dose. 
curve (Figure 2c) and using time for a given exposure to index the limits of integration. As a point of comparison, a relative dose of 55 corresponds to 95 seconds of source exposurc. The data of Figure 3 confirms the predicted behavior with an approximate linear response over the first 40 units of dose, followed by a significant decrease in slope between 40 and 60 relative units.

The presence of Cs on the sapphire surface can be confirmed using surface spectroscopy. Derivative mode AES spectra for Cs exposure times of 75, 90, and 120 seconds are shown in Figure 4. The Cs(MNN) transition is visible at $560 \mathrm{eV}$ and increases with exposure time. The Cs(NVV) transition overlaps the Al(LVV) and produces some significant changes in lineshape in the low kinetic energy region $(<60 \mathrm{eV})$ of the spectra. The substrate transitions remain the most predominant features in the spectra despite the fact that Cs exposure has passed the point of rapid decay in the sticking coefficient. These observations are consistent with limited Cs coverages produced in the initial adsorption regime probed by the deposition curves of Figure 2 and the desorption results of Figure 3 . The attenuation in the substrate $\mathrm{O}(\mathrm{KLL})$ signal can be used to estimate the Cs overlayer thickness at high exposures using the method of Seah and Dench (1979). The observed decrease of $26 \%$ corresponds to less than a one monolayer continuous film of Cs. Our limited investigation of the post-submonolayer deposition of Cs does not rule out the possibility of the formation of three dimensional islands of Cs on the sapphire surface. This growth mode has been observed for $\mathrm{Cs}$ on $\mathrm{NiO}(100)$ (Kennou et al. 1991) and oxidized W(110) (Desplat et ai. 1980).

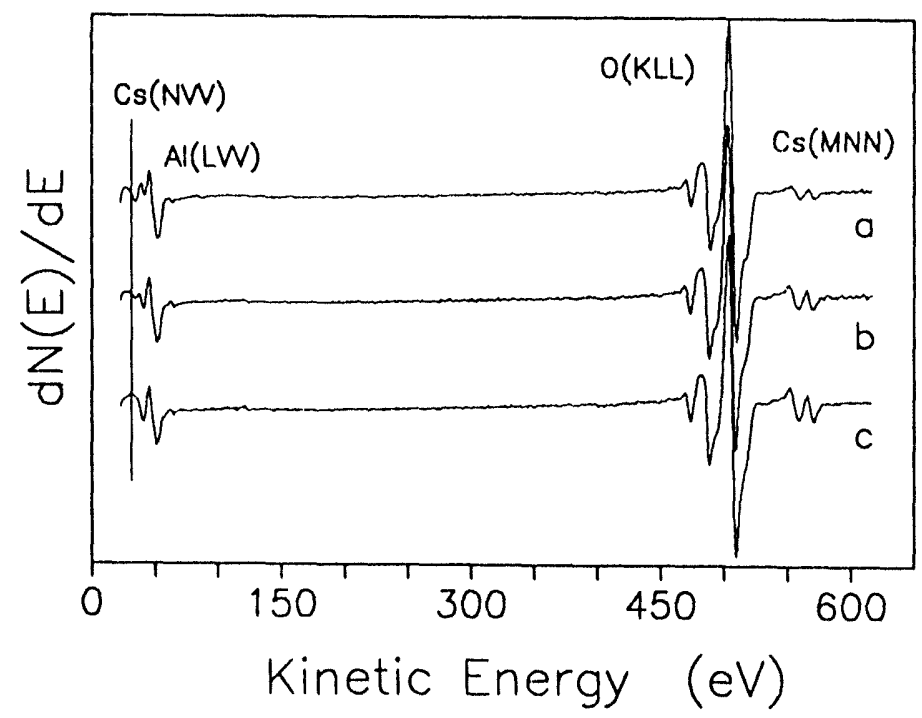

FIGURE 4: Variation in the Derivative Mode Auger Spectra for Sapphire with Increased Exposure to Cs for 75 (a), 90 (b), and 120 (c) Seconds of Source Exposure.

It is clear from the adsorption data presented above that the rapid decay in the initial sticking coefficient, demonstrated in the deposition profiles, occurs within the submonolayer coverage regime. The high sticking coefficient regime is of greatest interest for understanding how a sapphire surface might behave at the elevated temperatures and Cs partial pressures present in a thermionic device because of the enhancement in the adsorption energetics.

\section{Cs Desorution}

Thermal desorption mass spectrometric analysis of Cs on sapphire indicates that Cs is stabilized on the sapphire surface at high temperatures. Figure 5 shows a series of TDMS spectra for various Cs exposure times and relative dose values that correspond to pre-saturation of the surface. The TDMS data demonstrate that initial Cs exposure leads to the population of a high binding energy site characterized by a temperature for the maximum rate of desorption of $1290 \mathrm{~K}$. A feature at $850 \mathrm{~K}$ appears in tandem with the high temperature state and accounts for less 


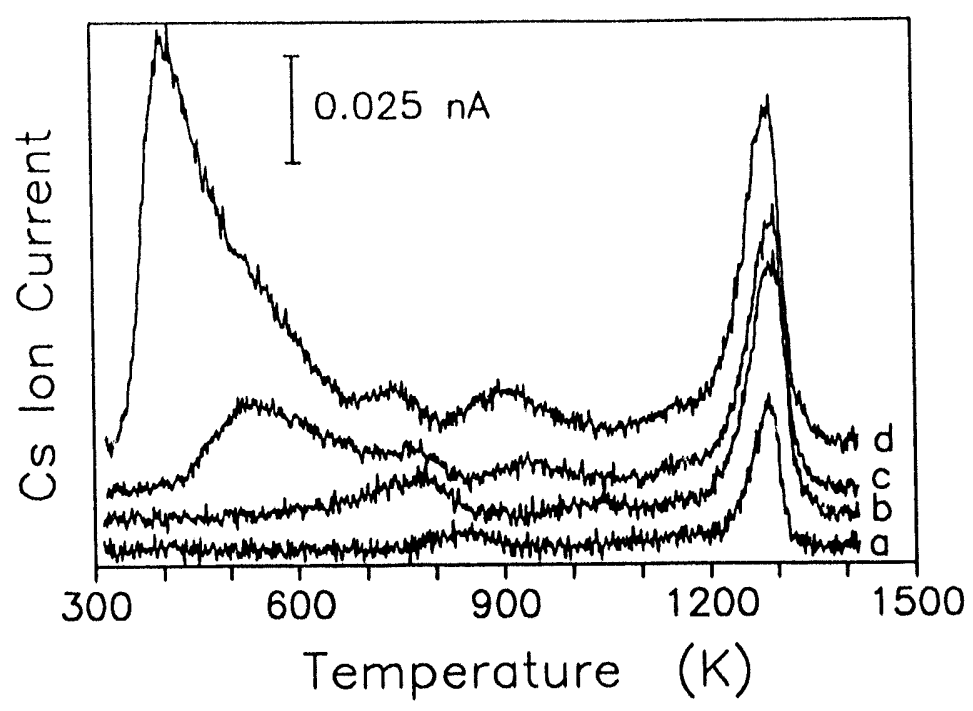

FIGURE 5: TDMS Spectra for Cs Deposited on Clean Sapphire at Relative Doses of 15 (a), 40 (b), 50 (c), and 80 (d).

than $5 \%$ of the total integrated Cs ion current. A series of additional low temperature features start to appear at 520 and $750 \mathrm{~K}$ as the high temperature feature approaches saturation. The peak maxima of these states shift to lower temperature with increased coverage, suggestive of Cs-Cs interactions playing a role in the desorption process. Post-saturation spectra (not shown) show additional increases in ion current in the lowest temperature state with a maximum rate of desorption occurring at $400 \mathrm{~K}$.

The TDMS spectra show significant variation in Cs desorption for different crystals. We have observed the two state behavior described above for three different crystals with some variation in the respective response of the low and high temperature states with exposure. At saturation, we have found that the high temperature state contributes 25 to $45 \%$ of the total integrated Cs signal. This observation indicates significant variation exists in the density of sites that yield tiis desorption state. We have also found that the degree of saturation of the high temperature state at which low temperature desorption first starts to occur can vary. This observation suggests that the energetics of access to these sites may vary significantly between surfaces. We believe that these variations are the result of differences in equilibrium surface structure between crystals. Chang (1971) has shown that a number of the low miller index sapphire surfaces undergo reconstruction in the 1300 to $1500 \mathrm{~K}$ range. Gautier et al. (1991) have demonstrated that the sapphire $(0001)$ surface actually passes through a series of surface structures on annealing from 1300 to $1700 \mathrm{~K}$. We presume that our randomly oriented surfaces assume some equilibrium surface structure at our maximum anneal temperature of $1500 \mathrm{~K}$.

Surface impurities appear to alter the adsorption and desorption characteristics of Cs. TDMS experiments conducted on sapphire with significant levels of a reduced-state of $C$ (see Figure 1b) show no trace of the high temperature state. Desorption profiles contain a single, broad desorption feature with a maximum desorption rate at $620 \mathrm{~K}$. The area under this feature is typically two to four times greater than that observed for the clean substrate at a constant Cs dose. The resulting AES spectra show relative increases in the Cs transitions with respect to the substrate, consistent with greater coverages for similar doses. The impact of a graphitic $C$ species at the surface appears to be the enhancement of Cs adsorption and stabilization of Cs on the sapphire surface to temperatures approximately $200 \mathrm{~K}$ greater than that observed for a clean sapphire surface.

We anticipate that the stabilization of Cs on sapphire in a TFE will depend on the relationship between surface composition and structure. It is difficult to separate these parameters because surface contaminants might drive 
changes in surface structure or compete for adsorption sites on a given structure. Cs stabilization to desorption temperatures greater than $1200 \mathrm{~K}$ would only be expected on sapphire in a TFE if surface structures are comparable at $900 \mathrm{~K}$ and $1500 \mathrm{~K}$ and $\mathrm{C}$ contamination is at a minimum. However, our results show that $\mathrm{C}$ remains on the surface at temperatures well in excess of $900 \mathrm{~K}$. We have also observed that Cs is not particularly effective at aiding in the removal of $\mathrm{C}$ from the sapphire surface at low Cs incident fluxes and low temperatures $(620 \mathrm{~K})$. Our experimental conditions are mild and may not adequately represent the operating conditions of a TOPAZ-II TFE. The presence of surface carbon alone raises concerns about changes in sapphire surface conductivity at elevated temperatures. This issue of the differences between compositionally and structurally driven changes and the impact on Cs adsorption/desorption requires further study.

\section{CONCLUSIONS}

The adsorption/desorption characteristics of $\mathrm{Cs}$ on clean sapphire surfaces have been successfully studied using a combination of surface analytical techniques. We have found that the initial adsorption of Cs on sapphire is quite facile with an approximate sticking coefficient of 0.9 . Cs adsorption slows significantly once a critical submonolayer coverage of $\mathrm{Cs}$ is achieved. TDMS analysis demonstrates that a high binding energy state, defined by a temperature for maximum rate of desorption of $1290 \mathrm{~K}$, fills first. Several low temperature states also start to fill in this initial adsorption regime. We believe that observed differences between crystals in this two state desorption behavior are the result of differences in the surface equilibrium structures produced at $1500 \mathrm{~K}$. Surface impurities, such as C, eliminate this high binding energy state of Cs on sapphire. However, C contaminants increase the sticking coefficient for Cs adsorption and delay the bulk of Cs desorption to temperatures greater than those found for the clean sapphire surface. Surface $C$ removal requires a temperature in excess of that achieved in a TFE environment, typically $900 \mathrm{~K}$ in a TOPAZ-II TFE. Whether Cs is stabilized on sapphire in a TFE environment will most likely depend on the relationship between surface contamination and surface structure.

\section{Acknowledaments}

The authors wish to acknowledge G. Cordes for technical assistance. This work was funded by the Ballistic Missile Defense Organization (BMDO) as part of the TOPAZ-II International Research Program. The work was performed at Phillips Laboratory and Sandia National Laboratories and supported by the U.S. Department of Energy under Contract No. DE-AC04-76DP00789.

$$
\begin{aligned}
& 94 A<85000 \\
& \text { References }
\end{aligned}
$$

Chang, C. C. (1971) "Silicon-on-Sapphire Epitaxy: LEED-Auger Studies and Electronic Properties of the Films," J. Vac. Sci. Technol., 8(3): 500-511.

Desplat, J.-L. and C. A. Papageorgopoulos (1980) "Interaction of Cesium and Oxygen on W(110), Part 1. Cesium Adsorption on Oxygenated and Oxidized W(110)," Surf. Sci., 92: 97-118.

Gautier, M., J. P. Duraud, L. Pham Van, and M. J. Guittet (1991) "Modifications of $\alpha-\mathrm{Al}_{2} \mathrm{O}_{3}(0001)$ Surfaces Induced by Thermal Treatments or lon Bombardment," Surf. Sci., 250: 71-80.

Hamza, A. V. and M. Balooch (1992) "The Adsorption of $\mathrm{C}_{60}$ and the Co-adsorption of $\mathrm{C}_{60}$ and $\mathrm{H}_{2} \mathrm{O}$ on $\alpha$ $\mathrm{Al}_{2} \mathrm{O}_{3}$ (1102)-(2x1)," Chem. Phys. Let., 198(6): 603-608.

Higgins, J. K. (1966) "Reaction of Alumina with Cacsium Vapour," Brit. Ceram. Soc. Trans., 65(12): 643-59.

Kennou, S., M. Kamaratos, and C. A. Papageorgopoulos (1991) "Cesium and Oxygen Adsorption on Ni(100)," Surf. Sci., 256: 312-316.

Seah, M. P. and W. A. Dench (1979), Surf. Interf. Anal. 1: 2. 

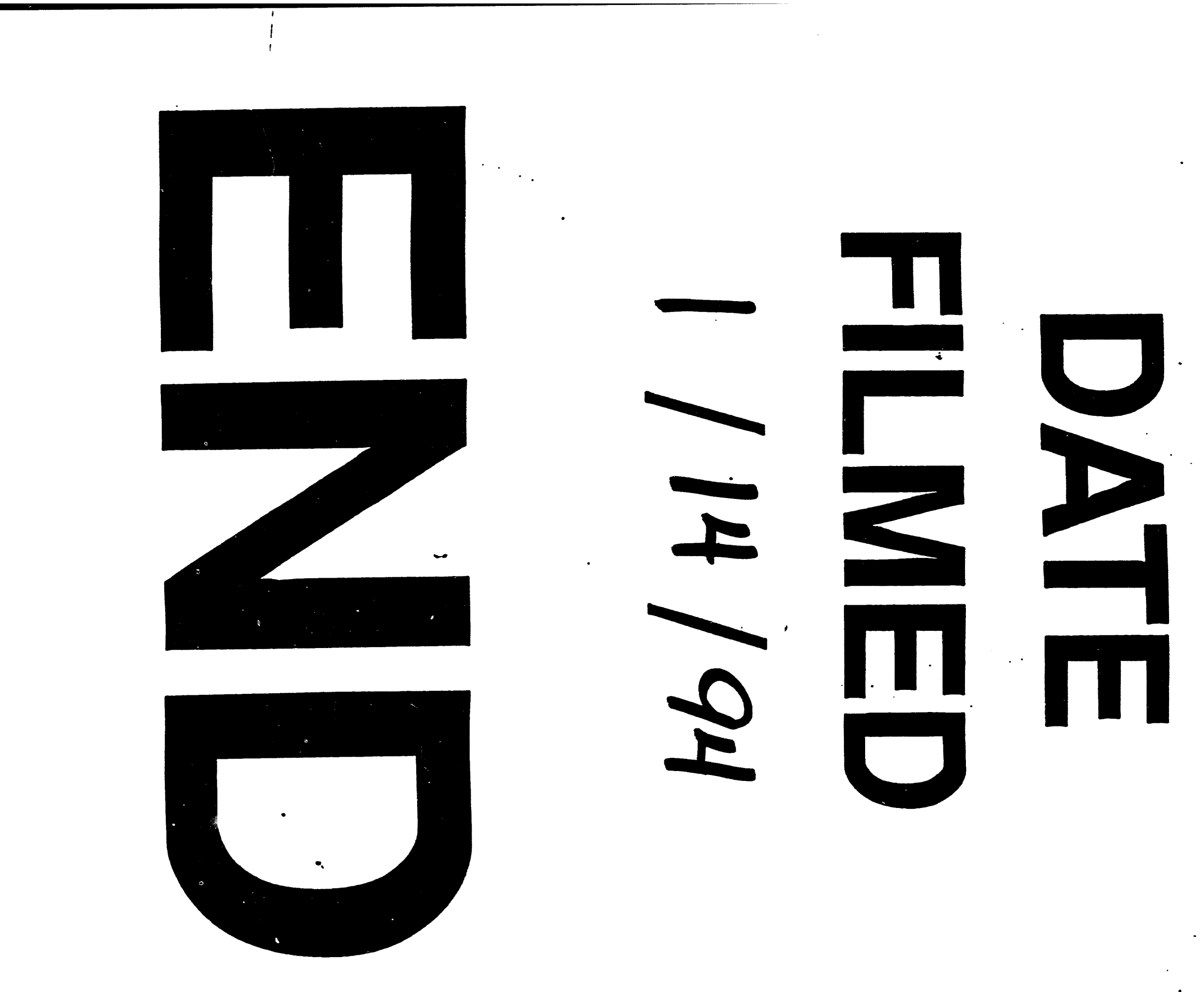


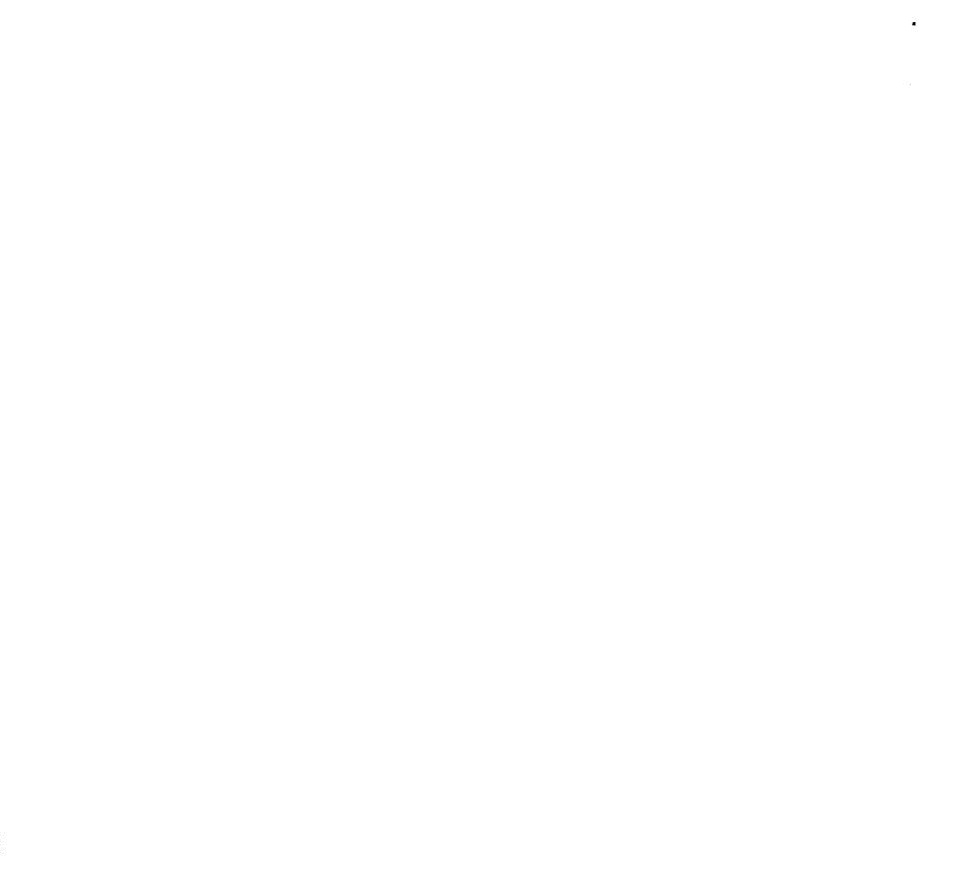

